the accurately known sidereal period of Mars, gave heliocentric directions and distances for a number of points on the martian orbit. And the last step, the one that commentators tend to feel more comfortable with, was to decide the nature of the curve determined by the points. The usual story is that Kepler found the curve to be an ellipse, to which the mention of 'perfect ellipse' in the title of Chapter 59 of New Astronomy gives credence. But with a proper measure of respect, this was an exaggeration. The martian orbit could be said to be an ellipse only to within the accuracy of Brahe's observations, and these were far from perfect.

Underlying every investigation by all the pioneers was the hypothesis that the planetary orbits (neglecting mutual interactions) were closed curves, requiring a unique relation between direction and distance as viewed by the Sun. It was just lucky that the pioneers did not have to cope with a world in which the post-newtonian relativistic approximation was important, when this hypothesis would have been untrue.

Quite possibly, Newton and Halley were aware of this point. Then one can readily understand the excitement over the orbits of comets of large eccentricity, which really did require the close perfection of the elliptic orbit. This matter of deep principle is surely the reason why comet Halley was so important to the newtonian world. For the perfect ellipse implied the inverse-square law of gravitation, and with that relationship firmly established, the march of science had become essentially unstoppable. It was in these books that it all began.

Sir Fred Hoyle is at 102 Admiral's Walk, West Cliff Road, Bournemouth, Dorset BH2 5HF, UK.

\section{The shape of things to come}

\author{
John L. Casti
}

Predicting the Future. Edited by Leo Howe and Alan Wain. Cambridge University Press: 1993. Pp. 195. E18.95, $\$ 29.95$.

WiThOUT too great an exaggeration, I think it's fair to say that the raison d'etre of intellectual endeavour in general art, literature, religion, music and all the rest - is to offer convincing answers to the eternal question: "Why do we see what we do and not see something else?" And science is no exception, being distinguished only by the form of the answers the scientist provides. The scientific answers come wrapped up in a set of rules, usually encapsulated in the form of a mathematical model or, more common nowadays, a computer program. And the purpose of these rules? Basically, to do two things: to explain past observations and to predict the results of new ones. So prediction and explanation are the twin pillars upon which the scientific enterprise rests. But, as this book makes clear, prediction and explanation are not concerns of the scientist alone, but lie at the centre of all types of intellectual life ranging from astrophysics to the Holy Scriptures.

Predicting the Future arose out of a series of lectures on the topic of "Predictions" given at Darwin College, Cambridge, in 1991. But from a glance at the table of contents, the volume might have been better titled "Predictions, Prophecies and Futurology". The book opens well enough, with thoughtful and informative accounts of the long-term be- haviour of the Universe by Stephen Hawking and the mysteries of chaotic dynamical processes by Ian Stewart. Both of these well-written essays are firmly centred in the scientific tradition of prediction by rule, showing that the rules we currently have at hand leave a lot to be desired when it comes to addressing the questions we most want to ask of nature.

Following a historical essay on the role of comets in prophecy through the ages by Simon Schaffer, the distinguished economist Frank Hahn offers probably the best account of the ins and outs of prediction in the book with his chapter on divining the mysteries of economic processes. Along the way to his almost foreordained conclusion that the predictive powers of economists are pretty feeble, Hahn brings out an important although often neglected aspect of prediction: namely, that not all predictions involve the future. He supports this claim with several convincing examples from the area of rational choice theory. Hahn ends with a general discussion of the possibility of effective predictions in the social sciences in general, a discussion that should sober up just about anyone infected with the germ of the idea that there's anything even remotely scientific about the social 'sciences'.

At this point, the book veers off the path of anything even faintly resembling prediction - scientific-style - flying off into the realms of futurology, historicism and prophecy. The first entry in this direction is an account of the possible future of medicine by Ian Kennedy. In pondering the medical frontier, Kennedy focuses on the bioethical dilemmas that the medical community is likely to face in coming years, including such things as the complex questions of care for an ageing population, how to handle developments in human genetics and the control of access to information about patients and their medical care.

The final three chapters abandon completely any pretence at prediction as that term is used in everyday speech, giving the floor over to religion, science's main competitor in the reality-generation game. Averil Cameron fires the opening salvo with his account of divine providence in late antiquity. This turns out to be a not very convincing attempt to show how late antiquity was characterized by a hierarchical Christian worldview that claimed to offer answers to a wide spectrum of human problems and was an infallible guide to predicting the future. The next chapter by Richard Gombrich on the role of prediction in the Buddhist scheme of things is a bit more satisfying, mostly because the author restricts his attention to a simple question: how open is the future from a Buddhist's perspective? Gombrich offers a threefold answer, in which the future can be both open and closed depending on whether or not an individual is one of the "enlightened'. Lastly, there is the Last Judgement, the theme of the book's final chapter by Don Cupitt. In this strange chapter, the author traces the history of the almost universal belief that there is a moral providence in the world ensuring that we will eventually get exactly what we deserve.

So what is one to make of this potpourri? To begin with, it's difficult to think of any single individual short of a reincarnation of Leonardo da Vinci whose intellect could simultaneously encompass the wide variety of views espoused here on the nature and effectiveness of prediction. Most scientists will find the second half of the book distinctly odd, if not completely eccentric, in treating prediction as more of a kind of religious and/or historical experience than as the working out of a set of scientific rules, or a computer progam, to get at the why and the when of things. All in all, this seems to be a volume more for browsing than for line-by-line reading. While the editors are to be commended for bringing such a disparate collection of views together within a single volume, it remains a volume in which the whole is somehow less than the sum of the parts.

John L. Casti is in the Institute for Econometrics, Operations Research and Systems Theory, Technical University of Vienna, Vienna, Austria, and the Santa $\mathrm{Fe}$ Institute, Santa $\mathrm{Fe}$, New Mexico 87501, USA. 